\title{
An improved experimental model for peripheral neuropathy in rats
}

\author{
Q.M. Dias, A.C. Rossaneis, R.S. Fais and W.A. Prado
}

Departamento de Farmacologia, Faculdade de Medicina de Ribeirão Preto, Universidade de São Paulo, Ribeirão Preto, SP, Brasil

\begin{abstract}
A modification of the Bennett and Xie chronic constriction injury model of peripheral painful neuropathy was developed in rats. Under tribromoethanol anesthesia, a single ligature with $100 \%$ cotton glace thread was placed around the right sciatic nerve proximal to its trifurcation. The change in the hind paw reflex threshold after mechanical stimulation observed with this modified model was compared to the change in threshold observed in rats subjected to the Bennett and Xie or the Kim and Chung spinal ligation models. The mechanical threshold was measured with an automated electronic von Frey apparatus $0,2,7$, and 14 days after surgery, and this threshold was compared to that measured in sham rats. All injury models produced significant hyperalgesia in the operated hind limb. The modified model produced mean \pm SD thresholds in $\mathrm{g}(19.98 \pm 3.08,14.98 \pm$ 1.86 , and $13.80 \pm 1.00$ at 2,7 , and 14 days after surgery, respectively) similar to those obtained with the spinal ligation model $(20.03 \pm 1.99,13.46 \pm 2.55$, and $12.46 \pm 2.38$ at 2, 7 , and 14 days after surgery, respectively), but less variable when compared to the Bennett and Xie model $(21.20 \pm 8.06,18.61 \pm 7.69$, and $18.76 \pm 6.46$ at 2,7 , and 14 days after surgery, respectively). The modified method required less surgical skill than the spinal nerve ligation model.
\end{abstract}

Key words: Chronic constriction injury; Chronic pain model; Neuropathy; Peripheral nerve lesion; Spinal nerve ligation

\section{Introduction}

Neuropathic pain can persist after nerve damage has been resolved and can be characterized as hypersensitivity to stimuli (hyperalgesia), the presence of abnormal sensations (hyperpathia) and a nociceptive response to non-noxious stimuli (allodynia) (1). Most studies show that the underlying pain mechanisms are associated with nerve injury, and analgesic alternatives for treating neuropathic pain can be evaluated using animal models of neuropathic pain (2).

Various experimental models can reproduce the neuropathic pain observed in human disease, and the ideal experimental model should result in reproducible sensory deficits, such as allodynia, hyperalgesia and spontaneous pain, over an extended period of time. The chronic constriction injury $(\mathrm{CCl})$ model was the first experimental model used (3), and involves 4 loose chromic gut ligatures placed around the sciatic nerve. However, the high levels of variability that may be observed in the data obtained with this model can be difficult to control when studying analgesic treatments for neuropathic pain (4). Alternatively, the spinal nerve ligation (SNL) model (5) involves a tight ligation of the L5 and L6 spinal nerves with silk thread. This model produces postoperative behaviors that indicate the pre- sence of neuropathic pain with minimal variability, but requires a high level of surgical skill.

In the present study, we describe a slight modification of the $\mathrm{CCl}$ method for producing painful peripheral mononeuropathy in rats. This modified model produces results with little variability when compared to results from the Bennett and Xie $\mathrm{CCl}$ model and requires less surgical skill than the Kim and Chung SNL model.

\section{Material and Methods}

\section{Animals}

Male Wistar rats $(140-160 \mathrm{~g})$ from the main animal house of the Ribeirão Preto Campus of the University of São Paulo were housed 2 to a cage and were maintained at a controlled temperature $\left(23 \pm 1^{\circ} \mathrm{C}\right)$, with free access to food and water. The experiments were approved by the Ethics Committee for Animal Research, Faculdade de Medicina de Ribeirão Preto, Universidade de São Paulo (No. 211/2005). The guidelines of the Committee for Research and Ethical Issues of the International Association for the Study of Pain were followed throughout the experiments (6). 


\section{Surgery}

Rats in the first group $(n=6)$ were anesthetized with tribromoethanol $(250 \mathrm{mg} / \mathrm{kg}$, ip) and subjected to the classic $\mathrm{CCl}$ model (3). After incision of the skin, the right sciatic nerve was exposed at the mid-thigh and freed from the adherent tissue proximal to its trifurcation. Four chromic gut loose ligatures (5-0) were located $1 \mathrm{~mm}$ apart and placed around the mid-sciatic nerve until the diameter of the nerve was slightly constricted just tightly enough to touch the nerve without interruption of epineural vasculature blood flow.

Rats in the second group $(n=6)$ were subjected to a slightly modified $\mathrm{CCl}$ model compared to that previously described (3). In this modified model, a single ligature of $100 \%$ cotton glace thread (Coats Corrente Ltda., Brazil) was placed around the right sciatic nerve proximal to its trifurcation until the diameter of the nerve was slightly constricted.

Rats in the third group $(n=6)$ were subjected to the SNL model of neuropathy. After incision of the skin, the right L5 and L6 spinal nerves were isolated and tightly ligated with a chromic catgut 5-0 suture, as described elsewhere (5). The incision was then closed with silk sutures by planes.

A group of 5 to 6 sham rats was used for each group of lesion-injured rats. The sham animals were subjected to similar procedures except for the nerve or spinal cord ligation. We used this procedure for all control rats. It differs from the Bennett and Xie's model regarding the use of non-operated paw threshold as control for the threshold of the operated paw. All rats were inspected daily during the first 14 postoperative days with respect to their gait, hind paw posture and signs of autotomy, as described elsewhere (3). Rats that exhibited motor deficits were excluded from further study.

\section{Algesimetric test}

The mechanical threshold for both hind paws of the rats was measured as previously described (7). The rat was placed in an acrylic cage $(12 \times 10 \times 17 \mathrm{~cm})$ with a wire-grid floor for approximately $30 \mathrm{~min}$ to allow for behavioral acclimation to the environment. The threshold for mechanical stimulation was measured with an automated electronic von Frey apparatus (IITC Electronic Equipment, USA), which consisted of a rigid plastic tip (tip area $=0.7 \mathrm{~mm}^{2}$ ) connected to a hand-held probe unit. A tilted mirror below the grid provided a clear view of the animal's hind paw. Pressure was applied to the plastic tip with increasing force in grams (g), in an upward direction against the central area of each hind paw. During this procedure, the applied force was continuously recorded by a main unit connected to the probe. The reflex threshold was determined by removal of the paw followed by clear flinching movements. After the rat withdrew its paw, the movement of the probe stopped, and the intensity of the pressure at the threshold was automati- cally recorded. A single trial consisted of 3 applications of the plastic tip, once every $5 \mathrm{~s}$ in each hind paw. The mean of 3 readings was taken as the threshold for a particular timing trial.

\section{Data analysis}

The threshold for hind paw reflex is reported as mean \pm SD. The reflex threshold was measured before the experiment (baseline) and 2, 7, and 14 days after surgery. Comparisons between the control (sham) and experimental groups at all time points were analyzed by multivariate analysis of variance (MANOVA) with repeated measures. The factors analyzed by MANOVA included treatment, time and treatment $x$ time interaction. In the case of a significant interaction (treatment $x$ time), a Bonferroni post hoc test was applied following the MANOVA. The analysis was performed using GraphPad Prism version 5.03 for Windows (GraphPad Software, USA). The level of significance was set at $P<0.05$.

\section{Results}

Animals subjected to the Bennett and Xie $\mathrm{CCl}$ neuropathy model (Figure $1 \mathrm{~A}$ ) had a mean hind paw reflex threshold $(\mathrm{g} \pm \mathrm{SD})$ at baseline that did not differ significantly from that of sham animals $(34.87 \pm 2.85$ versus $35.32 \pm 1.77$, respectively). The mean time of the surgical procedure was $20 \pm 2 \mathrm{~min}$. Two days after surgery, the mean reflex threshold was significantly lower in the lesion group $(21.20 \pm 8.06)$ compared to the sham group $(30.40 \pm 1.64)$. Seven and 14 days after surgery, the mean thresholds for the lesion group $(18.61 \pm 7.69$ and $18.76 \pm 6.46$, respectively) were lower than those for the sham group at the same times $(37.63 \pm 1.76$ and $38.31 \pm 1.11$, respectively). Two of the 6 rats in the lesion group $(40 \%)$ had thresholds very similar to the thresholds of the sham group 2 days after surgery. The variability in the thresholds decreased 7 and 14 days after surgery, but the thresholds of the 2 lesion-injured rats were still similar to those of the sham group. All lesion-injured rats had toes that were ventroflexed and walked by touching the floor with the medial edge of the hind paw. None of the rats exhibited signs of autotomy.

All the animals subjected to the SNL model (Figure 1B) also had a mean hind paw reflex threshold at baseline before surgery that did not significantly differ from that of the sham animals $(44.56 \pm 4.73$ versus $48.23 \pm 6.44$, respectively). The mean time of the surgical procedure was $56 \pm 6 \mathrm{~min}$. Two, 7 , and 14 days after surgery, all rats had thresholds lower than control. Two days after surgery, the mean reflex threshold was significantly lower in the lesion group $(20.03 \pm 1.99)$ compared to the sham group $(45.35 \pm 5.69)$. Seven and 14 days after surgery, the mean thresholds for the lesion group (13.46 \pm 2.55 and $12.46 \pm 2.38$, respectively) were lower than the thresholds for the sham group $(48.5 \pm 6.53$ and $49.35 \pm$ 

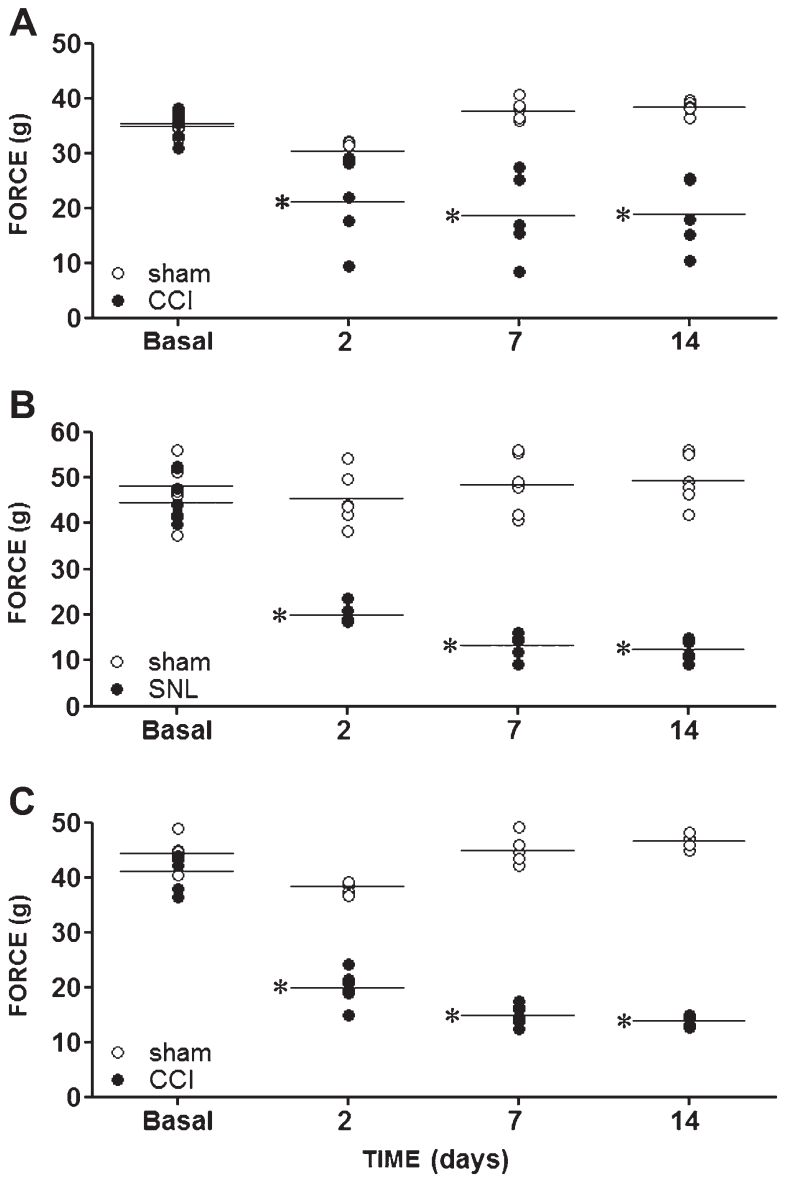

Figure 1. Time-course of hyperalgesia measured with an automated electronic von Frey apparatus. The changes in the reflex threshold of each rat are shown before surgery (basal) and 2,7 , and 14 days after chronic constriction injury $(\mathrm{CCl})$ of the sciatic nerve using the Bennett and Xie model $(A)$, sciatic nerve ligation (SNL) using the Kim and Chung model $(B)$, or a modified $\mathrm{CCl}$ model $(C)$. The horizontal bars indicate the mean threshold for each group of rats $(n=5-6)$. ${ }^{*} P<0.05$ compared to sham rats (MANOVA followed by the Bonferroni post hoc test).

5.29, respectively). The variability in the thresholds observed in the SNL group was minimal at all postoperative times. At no time point after surgery was the mean reflex threshold of the lesion-injured animals similar to that of the sham animals. All rats exhibited a variable degree of foot eversion on the lesion side, but none of the rats exhibited signs of autotomy.

All the animals subjected to the modified $\mathrm{CCl}$ model (Figure 1C) had a mean hind paw reflex threshold at baseline that did not differ significantly from that of the sham animals (41.00 \pm 3.00 versus $44.25 \pm 2.83$, respectively). The mean time of the surgical procedure was only $6 \pm 2 \mathrm{~min}$. Two, 7 , and 14 days after surgery, all rats had thresholds lower than control. Two days after surgery, the mean threshold of the lesion group (19.98 \pm
3.08) was significantly lower than that of the sham group $(38.28 \pm 1.00)$. Seven and 14 days after surgery, the mean thresholds were lower for the lesion group (14.98 \pm 1.86 and $13.80 \pm 1.00$, respectively) than for the sham group (44.76 \pm 2.48 and $46.58 \pm 1.14$, respectively). At no time after surgery was the mean reflex threshold of the lesion-injured animals similar to that of the sham animals. All rats held their toes together on the injured hind paw without showing signs of foot eversion or autotomy.

In all experimental groups, the reflex threshold to mechanical stimulation of the contralateral paw did not differ from its baseline threshold value (data not shown in the figure).

\section{Discussion}

The results of this study corroborate the $\mathrm{CCl}(3)$ and SNL (5) models of neuropathy that significantly reduce the reflex threshold of the injured hind paw in rats after mechanical stimulation. In addition, we showed that a minimally altered version of the Bennett and Xie $\mathrm{CCl}$ model can reduce the reflex threshold of the injured hind paw. Hyperalgesic responses to mechanical stimulation of the injured hind paw were evident 2, 7, and 14 days after operation in all models. However, the modified model appears to be advantageous in some aspects compared to the $\mathrm{CCl}$ and SNL models. The time required for surgery was less in the modified $\mathrm{CCl}$ model, which only required one ligature, compared to the Bennett and Xie $\mathrm{CCl}$ model. Moreover, the modified model produces reflex thresholds that are less variable than those produced with the Bennett and Xie $\mathrm{CCl}$ model. Actually, the standard deviations of the mean thresholds obtained with the Bennett and $\mathrm{Xie} \mathrm{CCl}$ were larger than those obtained with the modified $\mathrm{CCl}$ model at all experimental times. All animals submitted to the modified model developed significant reduction of the withdrawal threshold, whereas about $40 \%$ of the animals submitted to the Bennett and Xie $\mathrm{CCl}$ model did not have significant reduction of the thresholds compared to the thresholds of sham rats during the first 10 postoperative days.

The small variability noticed in the modified model may be explained by the physical characteristics of the cotton glace thread when compared to chrome catgut used in the $\mathrm{CCl}$ and SNL models. The high malleability of the cotton thread allows the sciatic nerve to be barely constricted by a ligature that does not move. The catgut thread has low malleability, so that ligatures are easily movable and quickly absorbed. The Bennett and Xie $\mathrm{CCl}$ model utilizes a slight nerve constriction and therefore the physical characteristics of the catgut affect the accuracy of the ligatures. This may be the reason why some animals subjected here to the Bennett and Xie $\mathrm{CCl}$ model did not display reduction of the mechanical threshold. The same limitation does not occur with the SNL model because in this case the spinal nerves are tightly constricted, thus 
allowing homogeneous reduction of reflex threshold. The cotton glace thread is coated with a starch-like substance, which may be the reason why a single ligature produced more effect than 4 ligatures of the same nerve.

The mean threshold and variability of the data obtained from individual SNL rats was very similar to the mean threshold of the modified $\mathrm{CCl}$ rats at all times after surgery. However, the surgical time was much longer for the SNL animals (56 $\pm 6 \mathrm{~min}$ ) compared to the $\mathrm{CCl}$ rats $(20 \pm 2 \mathrm{~min}$ in the Bennett and Xies model and $6 \pm 2 \mathrm{~min}$ in the modified $\mathrm{CCl}$ model), as a result of the deeper surgical planes required with the SNL model.

Behavioral changes, such as toe ventroflexion and walking with the medial edge of the hind paw, observed in the $\mathrm{CCl}$ group of rats were not different from the changes reported elsewhere (3). All rats in the SNL group showed variable degrees of hind paw inversion on the lesion side, as reported elsewhere (5). All rats in the modified $\mathrm{CCl}$ group only showed toes held together on the injured hind paw.

Compared to other models of sciatic nerve injury, the modified $\mathrm{CCl}$ method is the only model that utilizes a single ligature around the sciatic nerve. The Seltzer model utilizes a single ligation of one-third to one-half of the sciatic nerve with a silk thread (8). This model involves a partial denervation of the sciatic nerve after which neuropathic pain is particularly dependent on sympathetic tonus (9). A measurable and long-lasting reduction of the reflex threshold for mechanical stimulation is observed with the Seltzer model, but this reduction also occurs in the contralateral paw throughout the experimental time period. This observation differs from our data, which showed no observable change in the reflex threshold in

\section{References}

1. Treede RD, Jensen TS, Campbell JN, Cruccu G, Dostrovsky JO, Griffin JW, et al. Neuropathic pain: redefinition and a grading system for clinical and research purposes. Neurology 2008; 70: 1630-1635, doi: 10.1212/ 01.wnl.0000282763.29778.59.

2. Sorkin LS, Yaksh TL. Behavioral models of pain states evoked by physical injury to the peripheral nerve. Neurotherapeutics 2009; 6: 609-619, doi: 10.1016/ j.nurt.2009.07.007.

3. Bennett GJ, Xie YK. A peripheral mononeuropathy in rat that produces disorders of pain sensation like those seen in man. Pain 1988; 33: 87-107, doi: 10.1016/0304-3959(88)90209-6.

4. Sousa AM, Prado WA. The dual effect of a nitric oxide donor in nociception. Brain Res 2001; 897: 9-19, doi: 10.1016/ S0006-8993(01)01995-3.

5. Kim SH, Chung JM. An experimental model for peripheral neuropathy produced by segmental spinal nerve ligation in the rat. Pain 1992; 50: 355-363, doi: 10.1016/03043959(92)90041-9.

6. Zimmermann M. Ethical guidelines for investigations of the contralateral paw.

Another model of partial denervation is the spared nerve injury (SNI) model, which involves a lesion of two terminal branches of the sciatic nerve (tibial and common peroneal nerves) and leaves the remaining branch sural nerve intact (10). The SNI model differs from the SNL, $\mathrm{CCl}$ and Seltzer models because it allows the behavioral testing of skin in the non-injured territories adjacent to the denervated areas.

In order to reduce the number of rats to a minimum, the present results are based on a single test in each experimental group, in which a small number of animals was used. This may be the reason why the differences between the baseline in the first group (sham and $\mathrm{CCl}$ ) and the other two groups was as significant as the differences between the values observed in the sham and nervelesioned groups. However, other studies have also used a single test to validate their models: Li et al. (11) utilized only von Frey filaments, and Decosterd and Woolf (10) did not find changes in animal sensitivity to thermal stimulation while validating their SNI model of neuropathic pain.

The present study suggests that the modified $\mathrm{CCl}$ model is suitable for obtaining hyperalgesic responses to mechanical stimulation in rats. This modified model produced less variable data than those obtained using the Bennett and Xie $\mathrm{CCl}$ method and required less surgical skill compared to the SNL model.

\section{Acknowledgments}

Research supported by FAPESP (\#9/51258-2). Q.M. Dias, R.S. Fais and A.C. Rossaneis were recipients of FAPESP fellowships. experimental pain in conscious animals. Pain 1983; 16: 109110, doi: 10.1016/0304-3959(83)90201-4.

7. Prado WA, Dias TB. Postoperative analgesia induced by intrathecal neostigmine or bethanechol in rats. Clin Exp Pharmacol Physiol 2009; 36: 648-654, doi: 10.1111/j.14401681.2008.05128.x.

8. Seltzer Z, Dubner R, Shir Y. A novel behavioral model of neuropathic pain disorders produced in rats by partial sciatic nerve injury. Pain 1990; 43: 205-218, doi: 10.1016/03043959(90)91074-S.

9. Shir Y, Seltzer Z. Effects of sympathectomy in a model of causalgiform pain produced by partial sciatic nerve injury in rats. Pain 1991; 45: 309-320, doi: 10.1016/0304-3959(91)90056-4.

10. Decosterd I, Woolf CJ. Spared nerve injury: an animal model of persistent peripheral neuropathic pain. Pain 2000; 87: 149-158, doi: 10.1016/S0304-3959(00)00276-1.

11. Li D, Yang H, Meyerson BA, Linderoth B. Response to spinal cord stimulation in variants of the spared nerve injury pain model. Neurosci Lett 2006; 400: 115-120, doi: 10.1016/ j.neulet.2006.02.028. 